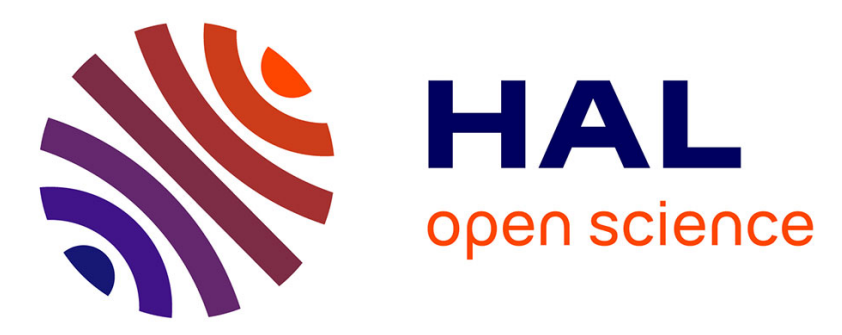

\title{
Explicit robustness margin for contractive piecewise affine control laws
}

\author{
Rajesh Koduri, Pedro Rodriguez-Ayerbe, Sorin Olaru, Morten Hovd
}

\section{To cite this version:}

Rajesh Koduri, Pedro Rodriguez-Ayerbe, Sorin Olaru, Morten Hovd. Explicit robustness margin for contractive piecewise affine control laws. 20th International Conference on System Theory, Control and Computing (ICSTCC), Oct 2016, Sinaia, Romania. pp.806 - 811, 10.1109/ICSTCC.2016.7790767 . hal-01429250

\section{HAL Id: hal-01429250 \\ https://hal-centralesupelec.archives-ouvertes.fr/hal-01429250}

Submitted on 25 Mar 2017

HAL is a multi-disciplinary open access archive for the deposit and dissemination of scientific research documents, whether they are published or not. The documents may come from teaching and research institutions in France or abroad, or from public or private research centers.
L'archive ouverte pluridisciplinaire HAL, est destinée au dépôt et à la diffusion de documents scientifiques de niveau recherche, publiés ou non, émanant des établissements d'enseignement et de recherche français ou étrangers, des laboratoires publics ou privés. 


\title{
Explicit robustness margin for contractive piecewise affine control laws
}

\author{
Rajesh Koduri ${ }^{1}$ Pedro Rodriguez-Ayerbe ${ }^{1}$ Sorin Olaru ${ }^{1}$ and Morten $\mathrm{Hovd}^{2}$
}

\begin{abstract}
This paper deals with the class of discrete-time linear dynamics affected by polytopic uncertainty in closed loop with contractive piecewise affine (PWA) control law. Starting from the hypothesis that the synthesis ensures a $\lambda$-contractive PWA control law for the nominal model, the objective is to calculate the robustness margin guaranteeing the contractivity (and consequently the asymptotic stability) of the closed loop dynamics. The robustness margin is represented as a subset of parameters within the set describing the polytopic uncertainty. For all these parameters, the $\lambda$-contractive behavior of the trajectories can be certified. This set of parameters is then compared to the set assuring the invariance of the considered closed-loop PWA dynamics which represents a limit case of the robustness study presented here.
\end{abstract}

\section{INTRODUCTION}

Model predictive control, possibly the most studied control law besides the conventional controllers, has the ability to handle constraints on inputs, states and outputs [7]. However, standard MPC can face computational difficulties for calculating the optimal control inputs in an iterative fashion especially when dealing with fast dynamics. To reduce the computational complexity faced by MPC, researches have come up with an Explicit MPC formulation [2], [1] in terms of a PWA control law. The idea of the Explicit MPC is to express the on-line quadratic problem in the control design into a multi-parametric Quadratic Programming (mp-QP) problem and to reduce the computation of the optimal control inputs to a simple evaluation of a control law stored in a look-up table. Such control laws can be easily implemented for realtime system with fast dynamics and relative small state-space models.

In the context of stability of the model predictive control, the terminal cost function and the terminal set is widely adapted in the problem formulation of the MPC at the design stage to ensure the state trajectories converge to the origin. A remarkable study on the control invariant characterization of linear systems is proposed in [4]. In this reference, the construction of successively tighter outer approximations for controlled $\lambda$-contractive set is obtained via an iterative algorithm. This approach was further extended to construct a noniterative controlled contractive set based on some conservative assumptions [5]. In this paper, we advocate the use of a

\footnotetext{
*This work is supported by TEMPO Marie-Curie European Project.

${ }^{1}$ The Authors are with L2S, CentraleSupelec, CNRS, UPS, Paris Saclay University, 91192, Gif-Sur-Yvette, France, ${ }^{2}$ The author is with the department of Engineering Cybernetics, NTNU, Norway. rajesh.koduri@centralesupelec.fr, pedro.rodriguezdcentralesupelec.fr,

sorin.olaru@centralesupelec.fr, morten.hovdeitk.ntnu.no.
}

controlled $\lambda$-contractive set that guarantees contractivity for each time step of the closed loop.

Starting from these framework, from the analysis point of view, it is important to commensurate the capacity of the control law to cope with disturbances, neglected dynamics or uncertain parameters. These characteristics is denoted in control theory as robustness of the controller. In the context of robustness analysis of PWA controllers, very few contributions have been made. Some noticeable recent works include an analysis procedure proposed in [8] and [11], which handles the robustness/fragility of the positive invariance for the dynamics affected by uncertain parameters. On the other hand, the robustness analysis can be connected to the works on the robustification of the explicit controllers. The reference [10] for example shows how to improve the robustness of the controller by retuning.

It is worth to be mentioned that the analysis of a nominal PWA control and its retuning is essentially different approach from a robust control design. It is known that a robust PWA control as for example robust explicit predictive control synthesis [12] can account for uncertainties based on dynamic programming approach but the associated computational complexity is exponential with respect to the nominal case. The same thing can be said about the robust explicit model predictive control with contractive set based on variable-structure control law for linear polytopic uncertain system as presented in [13].

In this paper, a multi-parametric quadratic programming problem is formulated in order to compute a PWA control law which enforces contractivity for the class of linear discrete-time systems. Starting from the $\lambda$-contractive control law, we present two robustness margins for the system stabilized by a contractive piecewise affine control law. First, the robustness margin for a discrete-time system affected by polytopic uncertainty is presented. The robustness margin denoted $\Omega_{r o b}$ and defined as a subset of the parametric uncertainty set $\Omega$ and is shown to take the form of polyhedral set. For all the models belonging to the polyhedral set $\Omega_{\text {rob }}$, the contractivity of the state trajectories are guaranteed in the presence of time-varying uncertainties. Second, we propose a numerical method to compute a gain margin set for a discrete-time system stabilized by a contractive PWA affine control. The desired gain margin takes the form of a set which characterizes admissible variations of system gains preserving the contractive characteristics of the controller.

The paper is organized as follow: First the definition of the basic notions on the explicit MPC is presented, and the 
properties of the $\lambda$-contractive explicit MPC are detailed. In section IV the construction of the $\lambda$-contractive controller via multi-parametric programming problem is presented. In section $\mathrm{V}$ the computation of robustness margin against parametric uncertainties is presented. The gain margin set for the contractive piecewise affine controller is shown in section VI. Examples at the end of sections V and VI illustrates the obtained robustness margins.

\section{Basic Notations AND PRELIMINARIES}

This section addresses some basic notations and definitions. A vector is defined as $x \in \mathbb{R}^{n}, x=\left[x_{1}, x_{2}, \cdots, x_{n}\right]^{T}$ and a matrix as $A \in \mathbb{R}^{n \times m}, A=\left[a_{i j}\right]$. An identity matrix is represented by $I_{n}$, where the subscript $n$ denotes the dimension of that matrix. The sets $\mathbb{R}, \mathbb{R}_{+}, \mathbb{Z}, \mathbb{N}$ and $\mathbb{N}_{+}$ denote set of real numbers, set of non-negative real numbers, set of integers, set of non-negative integers, set of positive integers respectively. We denote $\mathbb{R}^{n}$ a Euclidean space. Given a set $S$, we denote by $\mathcal{C} \operatorname{ard}(S)$ its cardinal number.

A set $S \subset \mathbb{R}^{n}$ is a proper $\mathcal{C}$-set if it is convex, closed, compact and contains the origin in its interior. A polyhedron is the (convex) intersection of a finite number of open or closed half-spaces and a polytope is a bounded and closed polyhedron. A mapping function $f: \mathbb{R}^{n} \rightarrow \mathbb{R}^{m}$ is said to be positively homogeneous of the first degree, if $f(\alpha x)=\alpha f(x)$, $\forall \alpha \in \mathbb{R}_{+}$and $\forall x \in \mathbb{R}^{n}$. The set of vertices of a polytope $M$ $\subset \mathbb{R}^{n}$ is denoted $\mathcal{V}(M)$. The unit simplex in $\mathbb{R}^{L}$ is defined as $\mathcal{S}_{L}=\left\{x \in \mathbb{R}_{+}^{L} \mid \mathbf{1}^{T} x=1\right\} . \mathbf{1}$ is a vector with all its elements equal to 1 . For a $N \in \mathbb{N}_{+}, \mathcal{I}_{N}$ denotes the set of integers, $\mathcal{I}_{N}:=\left\{i \in \mathbb{N}_{+} \mid i \leq N\right\}$. Let $P$ be a symmetric convex set in $\mathbb{R}^{n}$, then a function $\mathcal{M}_{p}(x)$ is a Minkowski function of $P$, if $\mathcal{M}_{p}(x)=\inf \left\{\gamma \in \mathbb{R}_{+}: x \in \gamma P\right\}$. $\operatorname{Proj}_{R^{c 2}} S$ is a projection mapping of a set $S$ onto a subset, $S \subset \mathbb{R}^{c 1} \rightarrow \mathbb{R}^{c 2}$ for $c 1>c 2$ and will be considered to operate on the first $c 2$ coordinates of $\mathbb{R}^{c 1}$. Finally, Conv denotes the convex hull.

\section{BACKGROUND AND OBJECTIVES}

Consider the discrete-time linear time-invariant system given by,

$$
x_{k+1}=A x_{k}+B u_{k}
$$

at all time instants $k \geq 0$. Here, $x_{k} \in \mathbb{R}^{n}$ is the state vector, $u_{k} \in \mathbb{R}^{m}$ is the control vector, $A \in \mathbb{R}^{n \times n}$ and $B \in \mathbb{R}^{n \times m}$. The system states and inputs variables are subject to constraints,

$$
\begin{array}{r}
X_{c}=\left\{x: H_{x} x \leq h_{x}, H_{x} \in \mathbb{R}^{n_{x} \times n}, h_{x} \in \mathbb{R}^{n_{x}}\right\} \\
U_{c}=\left\{u: H_{u} u \leq h_{u}, H_{u} \in \mathbb{R}^{m_{u} \times m}, h_{u} \in \mathbb{R}^{m_{u}}\right\},
\end{array}
$$

where the matrices $H_{x}, H_{u}$ and the column vectors $h_{x}, h_{u}$ are assumed to be constant, and $X_{c} \subset \mathbb{R}^{n}$ and $U_{c} \subset \mathbb{R}^{m}$. The state and input constraint sets $X_{c}$ and $U_{c}$ are polytopes containing the origin in their interior.

For the PWA control design, a quadratic cost of standard MPC with the terminal cost and stage cost is considered and subsequently transformed into a multi-parametric Quadratic
Programming (mp-QP) problem [2]. Considering the discretetime system in the form (1) subject to the constraints (2), the equivalent $\mathrm{mp}-\mathrm{QP}$ problem is written after algebraic manipulations as

$$
\begin{aligned}
& J\left(x, U^{*}\right)=\min _{U^{*}} \frac{1}{2} U^{* T} H U^{*}+x^{T} F^{T} U^{*}+\frac{1}{2} x^{T} Y x \\
& \text { s.t } \quad G U^{*} \leq D+S x \text {. }
\end{aligned}
$$

In (3), $x=x_{0}$, the column vector $U^{*}=$ $\left[u_{0}^{* T}, \cdots, u_{N_{p}-1}^{*}\right]^{T} \in \mathbb{R}^{m N_{p}}$ is the optimization vector and $N_{p}$ is the prediction horizon length. The matrices $H, F, Y, G, D, S$ are easily derived from the standard MPC cost (see [2] for the definition of the matrices and further details).

The optimal solution for (3) is represented by a finite set of affine functions defined over the polyhedral partition of the set $\mathcal{X}, \mathcal{X}=\cup_{i=1}^{N} \mathcal{X}_{i}$. The polyhedral $\mathcal{X}_{i} \subset \mathbb{R}^{n}$ are called critical regions or components of the partition. The polyhedral regions $\mathcal{X}_{i}$ are non overlapping i.e., $\operatorname{Int}\left(\mathcal{X}_{i}\right) \cap \operatorname{Int}\left(\mathcal{X}_{j}\right)=$ $\emptyset, \forall i \neq j$. Furthermore, two neighboring regions $\mathcal{X}_{i}$ and $\mathcal{X}_{j}$ share some vertices or facets. The proof of the properties of the mp-QP solution is presented in [2].

\section{Definition 1: A function}

$$
f_{\text {pwa }}:\left\{\mathbb{R}^{n} \rightarrow \mathbb{R}^{m} \mid A_{i} x+b_{i}, \forall x \in \mathcal{X}_{i}, i \in \mathcal{I}_{N}\right\}
$$

defined over the polyhedral partition of the set $\mathcal{X}$ is called a piecewise affine function of the polyhedral partition.

The explicit control law, solution of (3), is synthesized in terms of a continuous piecewise affine function defined over the polyhedral partition of the set $\mathcal{X}$ and it can be described by

$$
u_{p w a}\left(x_{k}\right)=F_{i} x_{k}+g_{i}, \quad \forall x_{k} \in \mathcal{X}_{i} .
$$

Definition 2: A closed and bounded set $\mathcal{P} \subseteq \mathcal{X}$ is called positively invariant with respect to the system (1) in closed loop with the control law $u_{\text {pwa }}\left(x_{k}\right)$ if for any $x_{0} \in \mathcal{P}$, it follows $x_{k} \in \mathcal{P}, \forall k \in \mathbb{N}_{+}$.

Considering the class of PWA feedback laws, we are interested in defining the notion of $\lambda$-contractiveness.

Definition 3: A $\mathcal{C}$-set $\mathcal{P} \subseteq \mathcal{X}$ is called controlled $\lambda$ contractive with contraction factor $\lambda \in[0,1)$ if and only if there exists an admissible control law $u_{p w a}\left(x_{k}\right)$ such that $\forall x_{k} \in \alpha P$ then $A x_{k}+B u_{p w a}\left(x_{k}\right) \in \lambda \alpha \mathcal{P}, \forall \alpha \in[0,1]$.

The controlled $\lambda$-contractive set represent an important notion that can be employed for stabilizing a constrained discrete-time linear systems. The properties of $\lambda$-contractive sets are enhanced versions of the positively invariant set. The polytopes generated from the contractive set are simpler than the reachable set and provide compact representations for the optimization based control design. 
In this paper, we consider parametric uncertainty on the $A$ and $B$ matrices of the system model (1). A set $\Omega$ is introduced in the parameter space,

$$
\Omega=\operatorname{Conv}\left\{\left[A_{1} B_{1}\right] \cdots\left[A_{L} B_{L}\right]\right\} .
$$

The nominal system is given by a convex combination,

$$
\left[\begin{array}{ll}
A & B
\end{array}\right]=\sum_{i=1}^{L} \zeta_{i}\left[A_{i} B_{i}\right]
$$

where $\zeta=\left[\zeta_{1}, \cdots, \zeta\right]^{T} \in \mathcal{S}_{L}$ and $\zeta_{i}$ is a non-negative scalar.

Definition 4: Consider a polytopic set $\Omega$, the robustness margin problem is to compute the largest subset $\Omega_{\text {rob }} \subset \Omega$ such that a given PWA control law $u_{\text {pwa }}(x)$ defined over the polyhedral set $\mathcal{X}$ for the system model (1) is controlled $\lambda$ contractive.

\section{PRELIMINARIES}

In the following, we introduce few matrix notations which will be used throughout the paper in relationship with the generators of the polytopic sets. Recall the polyhedral set $\mathcal{X}$, and its partition $\mathcal{X}=\cup_{i \in I_{N}} \mathcal{X}_{i}$. The set $\mathcal{X}$ and each of the regions composing the partition $\mathcal{X}_{i}$ are closed and bounded polyhedral sets and can be described by the vertex representation,

$$
\begin{gathered}
\mathcal{X}=\operatorname{Conv}\left\{v_{1}, v_{2}, \cdots, v_{r}\right\} \\
\mathcal{X}_{i}=\operatorname{Conv}\left\{w_{i 1}, w_{i 2}, \cdots, w_{i r_{i}}\right\}
\end{gathered}
$$

where $r$ and $r_{i}$, for $i \in \mathcal{I}_{N}$ denote the number of vertices for $\mathcal{X}$ and $\mathcal{X}_{i}$ respectively.

The vertex representation of the polytope $\mathcal{X}$ with the corresponding sets of vertices $\mathcal{V}(\mathcal{X})$ given in (8) define a finite subset of $\mathbb{R}^{n}$. Consequently, these vertices can be stored as columns of a matrix $W \in \mathbb{R}^{n \times r}$.

$$
W=\left[v_{1}, v_{2}, \cdots, v_{r}\right] .
$$

The vertices given in (9), form a different subset of vectors in $\mathbb{R}^{n}$. The vertices associated with each polytope $\mathcal{X}_{i}$ is referred as $\mathcal{V}\left(\mathcal{X}_{i}\right)$. It should be noted that a region and its neighboring region may have some vertices in common. Therefore, the vertices are arranged with a cardinal number $p$ $=\operatorname{Card}\left(\mathcal{V}\left(\mathcal{X}_{i}\right)\right)$, thus avoiding the column wise redundancies,

$$
V=\left[\mathcal{V}\left(\mathcal{X}_{i}\right)\right]=\left[w_{1}, w_{2}, \cdots, w_{p}\right]
$$

here $V \in \mathbb{R}^{n \times p}$. Finally, the knowledge of an admissible input for each vertex stored in the matrix $V$ allows the construction of a matrix $U \in \mathbb{R}^{m \times p}$,

$$
U=f_{p w a}(V)
$$

where the application of the function $f_{p w a}($.$) should be$ interpreted column wise.

\section{CONTROL DESIGN FOR CONTROLLED $\lambda$-CONTRACTIVE} SET

In this section, we discuss control design based on the explicit MPC law, but with an additional $\lambda$-contractive constraint imposed on the problem formulation. To facilitate an explicit control with controlled $\lambda$-contractive, it is desirable to compute an initial contractive set that does not violate the original state constraints. The controlled contractive sets of the shape specified in [5] are considered. To calculate an initial set, let us consider the system (1) and the state constraints, with a matrix $A=V_{A} D_{A} V_{A}^{-1}$. Here $V_{A}$ and $D_{A}$ denote the matrices of eigenvectors and diagonal eigenvalues matrix corresponding to the Jordan decomposition of matrix $A$. The obtained initial set is symmetric and can be represented as,

$$
\mathcal{P}=\left\{x:\left[\begin{array}{c}
V_{A}^{-1} \\
-V_{A}^{-1}
\end{array}\right] x \leq\left[\begin{array}{c}
k_{x} \\
k_{x}
\end{array}\right], V_{A} \in \mathbb{R}^{n \times n}, k_{x} \in \mathbb{R}^{n}\right\} .
$$

The contractiveness does not inherently require the set to be described in the form (13), it was just one approach to find a particularly simple controlled contractive set. The quadratic cost function for the controller stabilizing a linear discrete-time system given by (1) subject to constraints (2) is formulated as,

$$
\begin{array}{r}
J\left(x_{k}, U^{*}\right)=\min _{U^{*}} \sum_{i=1}^{N_{p}}\left\|Q x_{k+i}\right\|_{2}^{2}+\sum_{i=0}^{N_{p}-1}\left\|R u_{k+i}\right\|_{2}^{2} \\
\text { s.t. } \quad x_{k+1}=A x_{k}+B u_{k}, \quad k=0, \cdots, N_{p}-1 \\
x_{0}=x(0) \\
{\left[\begin{array}{c}
V_{A}^{-1} \\
\left.-V_{A}^{-1}\right]
\end{array}\right] x_{k+1} \leq \lambda \alpha\left[\begin{array}{l}
k_{x} \\
k_{x}
\end{array}\right]} \\
u_{k} \in U_{c}, k=0, \cdots, N_{p}-1 \\
x_{k} \in X_{c}, k=1, \cdots, N_{p}
\end{array}
$$

Here $\lambda$ is a pre-defined contractive factor, $\lambda \in[0,1)$ and $\alpha \in$ $[0,1]$ will be considered as a parameter. The weight matrices $Q=Q^{T} \geq 0$ and $R=R^{T}>0$ are positive semi-definite and positive definite respectively which define the performance index of the optimization problem (14)[7].

In (14a) we use an optimization criterion spanning over a multiple-time step horizon, while the contraction is only imposed for the first time step. Anyhow, the contractive set constraint in (14d) should be designed to make (14f) redundant.

Now, one has to transform the problem (14) into multiparametric programming problem including the full vector of parameters. This complete mp-QP problem is formulated for the state vector $x$ and $\alpha$ as an augmented parameter vector $\hat{x}$ $=[x, \alpha]^{T}$ leading to the compact cost formulation:

$$
J\left(U^{*}(\hat{x})\right)=\min _{U^{*}} \frac{1}{2} U^{* T} H U^{*}+\hat{x}^{T} F^{T} U^{*}+\frac{1}{2} \hat{x}^{T} Y \hat{x} .
$$

The constraints for the state and input variables can be appended to the inequality constraint given below,

$$
G U^{*} \leq D+S \hat{x}
$$


In (15) and (16), $\hat{x}=\hat{x_{0}}$. Subsequently, the initial contractive set given in (13) is also extended to $\hat{x}$ space, by setting the bounds of $\alpha$ parameter between 0 and 1. This set is herewith denoted as $\hat{\mathcal{P}} \in \mathbb{R}^{n+1}$. It inherits the polyhedral structure and will be included in the problem formulation via a set of linear constraints with a linear dependence of the right hand side on the extended parameter vector $\hat{x}$.

The contractive set $(14 d)$ represented by the inequality constraints can be written within (16):

$G=\left[\left[\begin{array}{c}V_{A}^{-1} \\ -V_{A}^{-1}\end{array}\right] * B,\left[\begin{array}{l}0 \\ 0\end{array}\right]_{2 n \times N_{p}-1}\right]$
$\left.S=\left[\begin{array}{c}-V_{A}^{-1} \\ V_{A}^{-1}\end{array}\right] * A, \lambda\left[\begin{array}{l}k_{x} \\ k_{x}\end{array}\right]\right]$ and $D=\left[\begin{array}{l}0 \\ 0\end{array}\right]_{2 n \times 1}$

From the problem formulation it can be noted that the state vector $x$ and $\alpha$ are the new parameters of the mp-QP problem. The state space partition obtained from the problem (15) and (16) can be represented as a finite collection of regions in the extended $[x, \alpha]$ space. This will be denoted next as

$$
\hat{\mathcal{X}}=\cup_{i \in \mathcal{I}_{\hat{N}}} \hat{\mathcal{X}}_{i}
$$

The control law obtained from the mp-QP formulation is given by,

$$
\hat{u}_{p w a}\left(\hat{x}_{k}\right)=\hat{F}_{i} \hat{x}_{k}+\hat{g}_{i}, i \in \mathcal{I}_{\hat{N}}
$$

In the following, we further explore the parametric dependence on $\alpha$ with the aim to reduce the extended vector [ $x$, $\alpha]$-space to the initial $x$-space by preserving the piecewise affine formulation. This can be done with a particular choice of $\alpha$ which can be interpreted as an implicit function of $x$ using in practice the Minkowski function $\mathcal{M}_{p}$ with respect to the initial contractive set $\mathcal{P}$. We recall here some of the basic properties.

Lemma 1: Let $\mathcal{P}$ be a convex set containing 0 as an interior point. Then the Minkowski function $\mathcal{M}_{p}$ of $\mathcal{P}$ satisfies:

1) $\mathcal{M}_{\mathcal{P}}$ is continuous,

2) $\mathcal{M}_{\mathcal{P}}$ is piecewise linear,

3) $\mathcal{M}_{\mathcal{P}}\left(x_{1}+x_{2}\right)=\mathcal{M}_{\mathcal{P}}\left(x_{1}\right)+\mathcal{M}_{\mathcal{P}}\left(x_{2}\right)$.

Now, introduce a subset $\mathcal{H} \subset \mathbb{R}^{n+1}$ defined as the graph of the Minkowski function with respect to the set $\mathcal{P} \subset \mathbb{R}^{n}$.

$$
\mathcal{H}=\left\{\left[\begin{array}{l}
x \\
\alpha
\end{array}\right]: \mathcal{M}_{\mathcal{P}}(x)=\alpha\right\}
$$

The PWA function (18) can be restricted to the subset $\mathcal{H} \cap \hat{\mathcal{X}}$ and subsequently projected onto the original state space. This results in an explicit PWA function:

$$
u_{p w a}\left(x_{k}\right)=F_{i} x_{k}+g_{i}, i \in \mathcal{I}_{N}, \text { for } x_{k} \in \mathcal{X}_{i}
$$

defined over a partition $\mathcal{X}, \mathcal{X}=\bigcup_{i \in \mathcal{I}_{N}} \mathcal{X}_{i}$. This design procedure is resumed in Algorithm 1.

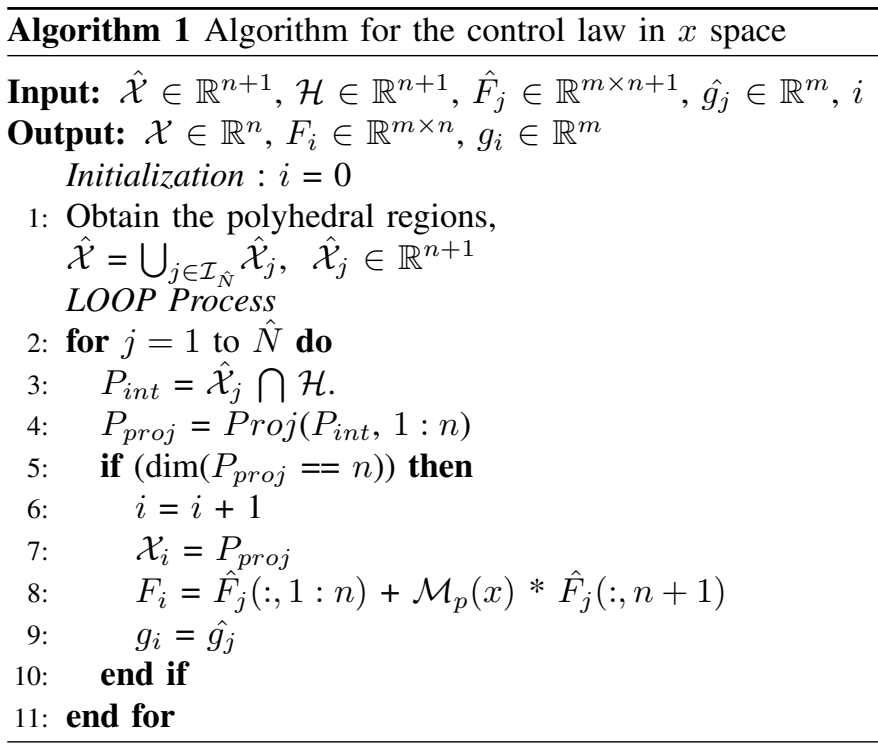

\section{ROBUSTNESS MARGIN FOR $\lambda$-CONTROLLED CONTRACTIVE SETS}

The robustness margin for the projected polyhedral set $\mathcal{X}$ can be constructed by using the vertex and half-space representation. In this paper we focus on the vertex representation and start by recalling the nominal system (1) subject to the constraint (2) used for the design of a contractive piecewise affine control law. The matrices $[A B]$ belong to the polytopic uncertain set $\Omega$ as defined in (6). The robustness margin problem is to compute the subset $\Omega_{r o b} \subset \Omega$ such that the closed loop dynamics obtained with the PWA control law defined over $\mathcal{X}$ is $\lambda$-contractive, that is:

$$
x_{k+1}=\left(A+B F_{i}\right) x_{k}+B g_{i} \in \lambda \alpha \mathcal{X}
$$

and $i \in \mathcal{I}_{N}$.

Let us define few important matrices which will be used in the following.

Recall the matrix $V$ defined in (11) which stores all the non-identical vertices of the $\mathcal{X}_{i}$ with a cardinal number $p$. The value of $\alpha$ parameter is computed for each column vector in the matrix $V$,

$$
\alpha_{i}\left(w_{i}\right)=\mathcal{M}_{\mathcal{P}}\left(w_{i}\right), i \in \mathcal{I}_{p}
$$

It is possible to express (21) as a convex combination of the vertices of the polyhedral set $\mathcal{X}$,

$$
\sum_{j=1}^{r} \gamma_{j} v_{j}, \gamma=\left[\gamma_{1}, \cdots, \gamma_{r}\right]^{T} \in \mathcal{S}_{r}, \mathbf{1}^{T} \gamma=1
$$

Rewriting (21) we obtain:

$$
\left(A+B F_{i}\right) x_{k}+B g_{i}=\lambda \alpha\left(x_{k}\right) W \gamma_{k} .
$$

Replacing (22) within (23) one can compute for each column vector in the matrix $V$ with the corresponding vector $\gamma_{i}$. Storing the column vectors $\gamma_{i}, i=1, \ldots, p$, a matrix $\Gamma \in \mathbb{R}^{r \times p}$ will be obtained

$$
\Gamma=\left[\alpha_{1} \gamma_{1}, \alpha_{2} \gamma_{2}, \cdots, \alpha_{p} \gamma_{p}\right]
$$


Finally, after defining a matrix $M \in \mathbb{R}^{n \times r}$ as a simple scaling of the vertices of the feasible set $M=\lambda W$ one can state the main result with respect to the robustness margin characterization.

Theorem 1: Consider a discrete-time system (1) subject to a polytopic uncertainty and subject to the states and input constraints. The robustness margin for a given contractive piecewise affine control law is given by

$$
\Omega_{\text {rob }}=\operatorname{Proj}_{\mathcal{S}_{L}} \mathcal{T}
$$

where $\mathcal{T}$ represents the polyhedral set,

$$
\mathcal{T}=\left\{\begin{array}{c}
\left\{(\zeta, \Gamma) \in \mathcal{S}_{L} \times \mathbb{R}^{r \times p} \mid \mathbf{1}^{T} \Gamma=\left[\alpha_{1}, \cdots, \alpha_{p}\right],\right. \\
\left.\sum_{j=1}^{L} \zeta_{j}\left(A_{j} V+B_{j} U\right)=M \Gamma\right\} .
\end{array}\right\} .
$$

Proof: To prove the existence of robustness margin for the polyhedral set $\mathcal{X}$ whose control law is associated with controlled $\lambda$-contractive set, let us consider the closed loop formulation of the piecewise affine control law with the $\lambda$-contraction.

$$
\left(A+B F_{i}\right) x+B g_{i} \in \lambda \alpha \mathcal{X}
$$

where $\lambda$ denotes the contractive factor and the parameter $\alpha(x)=\mathcal{M}_{\mathcal{P}}(x)$. We recall that $\forall(A, B) \in \Omega_{\text {rob }}, \forall x \in \mathcal{X}_{i}$, $\forall i \in \mathcal{I}_{N}$ and considering the polytopic uncertainty set $\Omega$ we can show that $\Omega_{r o b} \subset \Omega$. And clearly, (27) can be written as

$$
\sum_{j=1}^{L} \zeta_{j}\left(A_{j}+B_{j} F_{i}\right) x+\zeta_{j} B_{j} g_{i} \in \lambda \alpha \mathcal{X} .
$$

Now, simply the state vector, $x \in \mathcal{X}_{i}$, can be expressed as a convex combination of the vertices, $x=\sum_{l=1}^{r_{i}} \beta_{l} w_{i l}$ for $\sum_{l=1}^{r_{i}}$ $\beta_{l}=1$, with $\zeta_{j}$ the elements of a vector $\zeta \in \mathcal{S}_{L}, \forall i \in \mathcal{I}_{N}$ and $\forall l \in \mathcal{I}_{r_{i}}$.

Subsequently replacing $x$ with $w_{i l}$, the $\alpha$ parameter for the vertex $w_{i l}$ is computed by $\alpha_{i l}=\mathcal{M}_{\mathcal{P}}\left(w_{i l}\right)$. Equivalently $(28)$ is followed by,

$$
\sum_{j=1}^{L} \zeta_{j}\left(A_{j}+B_{j} F_{i}\right) w_{i l}+\zeta_{j} B_{j} g_{i} \in \lambda \alpha_{i l} \mathcal{X}
$$

Moreover, the inclusion can be explicitly described by the existence of $y_{i l} \in \lambda \alpha_{i l} \mathcal{X}$ such that:

$$
\sum_{j=1}^{L} \zeta_{j}\left(A_{j}+B_{j} F_{i}\right) w_{i l}+\zeta_{j} B_{j} g_{i}=y_{i l} .
$$

The vector $y_{i l} \in \mathbb{R}^{n}$ can be expressed as,

$$
y_{i l}=\lambda \alpha_{i l}[\mathcal{V}(\mathcal{X})] \gamma_{i l} \quad \text { for } \quad \gamma_{i l} \in \mathcal{S}_{r}
$$

Substituting (31) in (30) and introducing a matrix $M \in$ $\mathbb{R}^{n \times r}$, where $M=\lambda W$ we obtain,

$$
\sum_{j=1}^{L} \zeta_{j}\left(A_{j}+B_{j} F_{i}\right) w_{i l}+\zeta_{j} B_{j} g_{i}=M \alpha_{i l} \gamma_{i l}
$$

From (32) it can be stated that if it holds $\forall l \in \mathcal{I}_{r_{i}}$, consequently it will hold for all the columns of the matrix $V$ as given in (11). Exploiting the admissible input mapping of the columns of $V$ as in $U$ leads to the matrix formulation,

$$
\sum_{j=1}^{L} \zeta_{j} A_{j} V+\zeta_{j} B_{j} U=M \Gamma
$$

It can be noticed that $\gamma_{i l} \in \mathcal{S}_{r}$, that is, each column of matrix $\Gamma$ is restricted to the simplex $\mathcal{S}_{r}$ multiplied by $\alpha_{i}$, $\forall i \in \mathcal{I}_{p}$. The above derivations prove that the polyhedral set $\mathcal{T}$ in (26) represents a parametrized set of all the model uncertainties guaranteeing the controlled $\lambda$-contractivity of the closed loop for the dynamical system affected by uncertainties. Further the polyhedral set $\mathcal{T}$ is projected on the simplex function $\mathcal{S}_{L}$.

\section{A. Example}

Consider a discrete-time linear system constructed from the uncertainty set described by:

$$
\Omega=\operatorname{conv}\left\{\begin{array}{l}
{\left[\begin{array}{ll}
A_{1} & B_{1}
\end{array}\right]=\left[\begin{array}{ccc}
0.4546 & -0.0913 & 0.0849 \\
0.1836 & 0.5389 & 0.0064
\end{array}\right],} \\
{\left[\begin{array}{ll}
A_{2} & B_{2}
\end{array}\right]=\left[\begin{array}{ccc}
0.7326 & -0.0767 & 0.0609 \\
0.1557 & 0.9909 & 0.0114
\end{array}\right],} \\
{\left[\begin{array}{ll}
A_{3} & B_{3}
\end{array}\right]=\left[\begin{array}{ccc}
1.0866 & -0.0861 & 0.0823 \\
0.1722 & 1.4323 & 0.0076
\end{array}\right]}
\end{array}\right\}
$$

with the nominal model chosen to be:

$$
x_{k+1}=A x_{k}+B u_{k}
$$

with $A=0.3 A_{1}+0.2 A_{2}+0.5 A_{3}$ and $B=0.3 B_{1}+0.2 B_{2}$ $+0.5 B_{3}$.

The input constraint is given by $-2 \leq u_{k} \leq 2$ and the state constraints are $-100 \leq\left[\begin{array}{ll}0 & 1\end{array}\right] x_{k} \leq 100$. The contractive factor $\lambda$ chosen is 0.99 . Unity weights are applied on the inputs and states penalties and the prediction horizon chosen is 2 . MultiParametric Toolbox is used to obtain the state space partition [9].

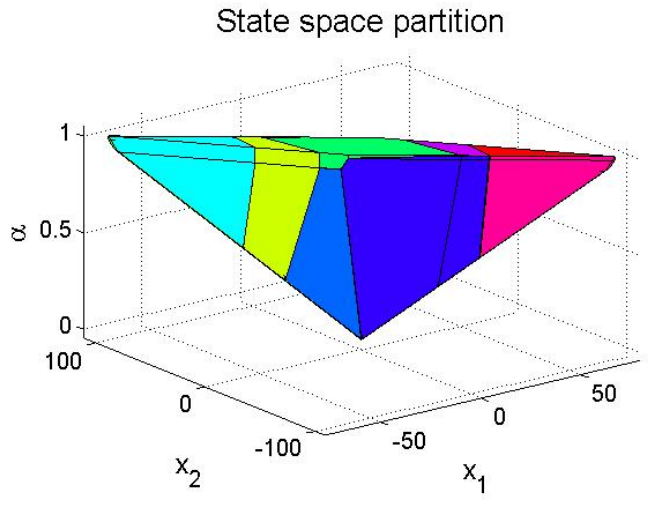

Fig. 1: State space partition with $\alpha$ as parameter 
Fig. 1, shows the state space partition of the above system and it has 11 regions. Fig. 2, shows the regions of the initial contractive set with the projected states $\left[x_{1}, x_{2}\right]$. Here the initial contractive set is divided into four regions and the hyperplane for the $\alpha$ parameter is calculated for each region. The projection is done using the proposed algorithm in section IV.

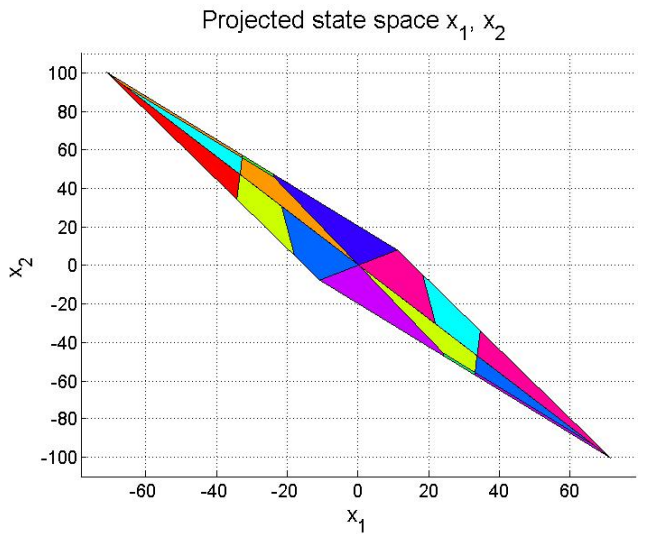

Fig. 2: Projected state space partition $x_{1}, x_{2}$.

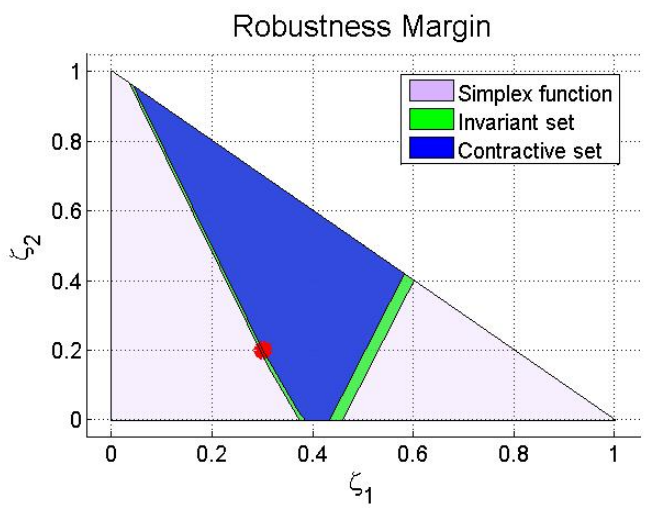

Fig. 3: Robustness margin for contractive and invariant set in the plane of $\zeta_{1}, \zeta_{2}$.

Fig. 3, shows the robustness margin $\Omega_{r o b}$ for the controlled $\lambda$-contractive set presented by the blue polytope and robustness margin for the controlled positively invariant set by the green polytope. The red dot denotes the considered nominal system where $\zeta_{1}=0.3$ and $\zeta_{2}=0.2$. For simplicity, the simplex function is presented only for $\zeta_{1}$ and $\zeta_{2}$ such that $\zeta_{3}=1$ $\zeta_{1}-\zeta_{2}$. It is observed that the given control law guarantees the contractivity of the feasible region $\mathcal{X}$ only if the system is inside the blue polytope. Similarly, the control law cannot guarantee either the positive invariance or contractivity of $\mathcal{X}$ if the system is away from the blue and green polytopes. For system inside the green polytope and outside the blue polytope the control law guarantees the invariance of the operating region $\mathcal{X}$. Fig. 4, shows the state trajectories for the same initial state $x_{0}=[-7098]^{T}$ for different nominal systems.

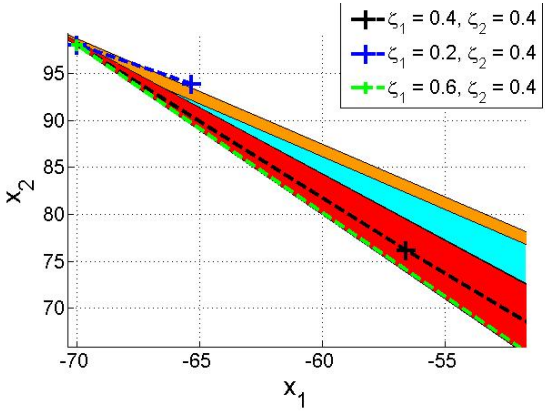

Fig. 4: Trajectories for different nominal systems for the same initial state.

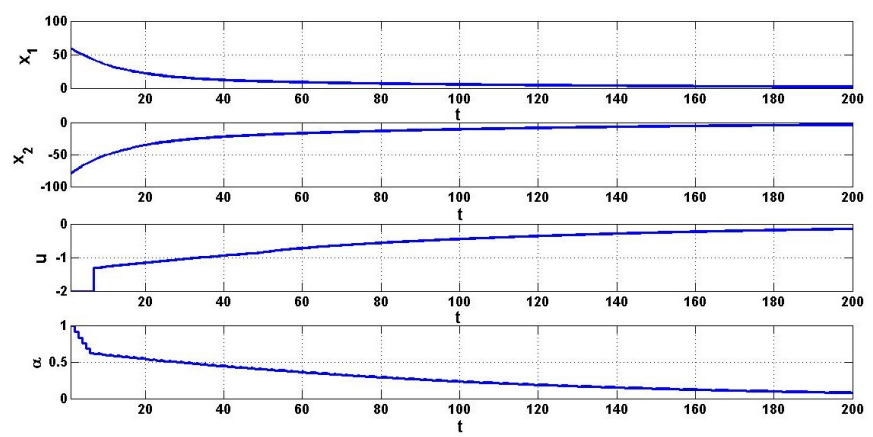

Fig. 5: Simulation for the state trajectories and control input for an initial state $x_{0}=\left[\begin{array}{ll}60-80\end{array}\right]^{T}$.

Fig. 5, depicts the simulation for the state trajectories and control input for a nominal system with $\zeta_{1}=0.3, \zeta_{2}=0.2$ and $\zeta_{3}=0.5$ and, for an initial state $x_{0}=\left[\begin{array}{ll}60 & -80\end{array}\right]^{T}$.

\section{Gain MARGin For $\lambda$-CONTROLLED CONTRACTIVE SETS}

In the following, we describe an analysis of the gain margin set for the system (1) stabilized with the help of a state feedback contractive PWA control law. The construction of the gain margin is similar with the one presented in the previous section and the proofs will be omitted.

Definition 5: Consider a discrete time linear system (1) with a continuous contractive PWA control law, such that the state space set $\mathcal{X}$ is controlled $\lambda$-contractive. The Gain Margin is represented by the set $\mathcal{K} \subset \mathbb{R}^{m}$, such that $x_{k+1}=A x_{k}+B\left(I_{m}+\operatorname{diag}\left(\delta_{K}\right)\right) u_{p w a}\left(x_{k}\right) \in \mathcal{X}, \forall x_{k} \in \mathcal{X}$ and $\delta_{K} \in \mathcal{K}, \delta_{K} \in \mathbb{R}^{m}$.

The set $\mathcal{K} \subset \mathbb{R}^{m}$ is a set which contains the input channels gain variations $\delta_{K}$ such that for any point inside the set $\mathcal{K}$, the $\lambda$-contractive characteristics of the set $\mathcal{X}$ is preserved. 
Theorem 2: Consider a discrete-time linear system (1) with a contractive piecewise affine state feedback control. The gain margin $\mathcal{K}$ of the controller is defined by the set,

$$
\mathcal{K}=\cap_{q=1}^{p} \mathcal{K}_{q},
$$

where $\mathcal{K}$ represents the gain margin set and $\mathcal{K}_{q}$ the local gain margin for the vertex $w_{q}$ for some $q \in \mathcal{I}_{p}$.

$$
\mathcal{K}_{q}=\left\{z \in \mathbb{R}^{m} \mid \exists u \in \Delta \mathcal{U}_{q}, M z=u\right\}
$$

with

$$
\Delta \mathcal{U}_{q}=\left\{u \mid \hat{H}_{u} u \leq \hat{h}_{u}\right\}
$$

where $\hat{H}_{u} \in \mathbb{R}^{d_{u} \times m}$ and $\hat{h}_{u} \in \mathbb{R}^{d_{u}} . \Delta \mathcal{U}_{q}$ represents the set of admissible input variation for the vertex $w_{q}$ preserving the contractivity.

The collection of sets $\mathcal{K}_{q}$ are independent for each vertex of the set in $\mathcal{X}_{i}$ and the intersection of these independent set gives the global set $\mathcal{K}$. In order to compute $\mathcal{K}_{q}$, we first need to compute explicitly the sets $\Delta \mathcal{U}_{q}$.

Theorem 3: Consider a linear discrete-time system (1) stabilized by a contractive piecewise affine control law. The set $\Delta \mathcal{U}_{q}$ of admissible input variations at the vertex $w_{q}$ is obtained by

$$
\Delta \mathcal{U}_{q}=\operatorname{Proj}_{U_{c}} \mathcal{H}_{q}
$$

$U_{c} \in \mathbb{R}^{m}$ denotes the input constraint set. The polyhedral set $\mathcal{H}_{q}$ is described by:

$$
\mathcal{H}_{q}=\left\{\left(\delta_{u}, \gamma\right) \in \mathbb{R}^{m} \times \mathbb{R}^{r}, \text { and }[A B]\left[\begin{array}{c}
x_{k} \\
u_{p w a}\left(x_{k}\right)
\end{array}\right]\right\}
$$

Proof: The proof is similar to the one presented in the previous section.

\section{A. Example}

Consider a discrete-time linear system,

$$
x_{k+1}=\left[\begin{array}{ll}
0.9 & 0.5 \\
0.2 & 0.8
\end{array}\right] x_{k}+\left[\begin{array}{l}
1.0 \\
0.2
\end{array}\right] u_{k}
$$

The input constraint is given by $-2 \leq u_{k} \leq 2$ and state constraint by $-5 \leq\left[\begin{array}{ll}0 & 1\end{array}\right] x_{k} \leq 5$. The prediction horizon chosen is 2 and unity weights are applied on the inputs and states penalties. The contraction factor $\lambda=0.98$ is considered.

The gain margin set $\mathcal{K}$ in (35) is computed for the PWA control law assuring the contractivity characteristics of the controller. As a term of comparison, with respect to the invariance, the value of $\delta_{K}$ lies between $[-0.524,1.554]$ while for the contractive controller, the value of $\delta_{K}$ lies between $[-0.467,1.445]$. Fig. 6 shows the projected state partition on the $x$-plane with state simulations in closed loop for an initial state $x_{0}=\left[\begin{array}{lll}0.355 & 5.0\end{array}\right]^{T}$ for different $\delta_{K}$ values.

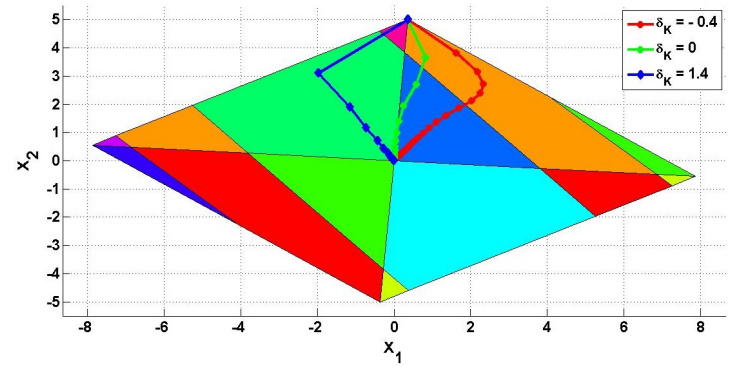

Fig. 6: Projected state space partition with state trajectories for different $\delta_{K}$ values.

State trajectories, control inputs and $\alpha$ values are simulated in closed loop for an initial state $x_{0}=[0.3555 .0]^{T}$ with different $\delta_{K}$ values, such that $x_{k+1}=A x_{k}+B\left(1+\delta_{K}\right) u_{p w a}\left(x_{k}\right)$, for the contractive controller and it is shown in Fig. 7. It is observed that the trajectories are $\lambda$-contractive thus confirming the theoretical result.

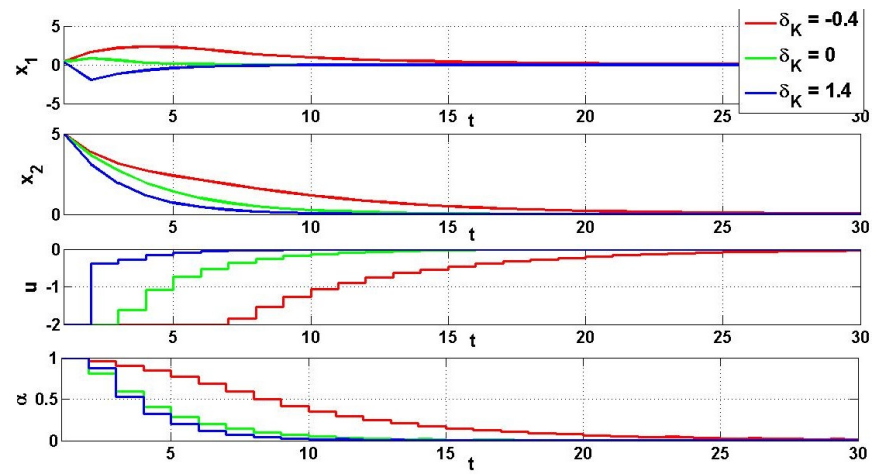

Fig. 7: Simulation for state trajectories, control input and $\alpha$ for different $\delta_{K}$ values.

\section{CONCLUSIONS}

In this work the robustness margin assuring the contractivity of trajectories for a linear uncertain system controlled by a piecewise affine control law has been deduced. Starting with a nominal contractive PWA control law, this robustness margin consists in a subset of the uncertain polytopic description of the plant. For all the systems described in this set the trajectories are contractive. Moreover a gain robustness margin have also been computed.

\section{REFERENCES}

[1] A. Alessio and A. Bemporad, A survey on explicit model predictive control, In Nonlinear model predictive control: Springer 2009, pp. $345-$ 369.

[2] A. Bemporard, M. Morrari, V. Dua and E. N. Pistikopoulos, The explicit linear quadratic regulator for constrained systems, Automatica, vol 38(1), pp. 3-20, 2002,

[3] G. C. Goodwin, H. Kong, G. Mirzaeva, and M. M. Seron, Robust model predictive control: reflections and opportunities, Journal of Control and Decision, vol. 1(2), pp. 115-148, 2014.

[4] C. Dorea and J. C. Hennet, $(a, b)$-invariant polyhedral sets for linear discrete-time systems, Journal of Optimization Theory and Applications, vol. 103(3), pp. 521-542, 2005.

[5] M. Hovd, S. Olaru and G. Bitsoris, Low Complexity constraint control using contractive sets), In World congress, vol.19, pp. 2933-2938, 2014 
[6] E. C. Kerrigan and J. M. Maciejowski, Robustly stable feedback min-max model predictive control, In American Control Conference, Proceedings of the 2003, vol. 4, pp. 3490-3495, 2003.

[7] D. Q. Mayne, J. B. Rawlings, C. V. Rao, and P. O. M. Scokaert, Constrained model predictive control: Stability and optimality, Automatica, vol. 36(6), pp. 789-814, 2000

[8] S. Olaru, N. A. Nguyen, G. Bitsoris, P. Rodriguez-Ayerbe and M. Hovd, Explicit robustness margins for discrete-time linear systems with PWA control, System Theory, Control and Computing (ICSTCC), 2013 17th International Conference, pp. 380-385, Oct. 2013

[9] M. Herceg and M. Kvasnica and C. N. Jones and M. Morari, MultiParametric Toolbox 3.0, Proc. of the European Control Conference, pp. 502-510, July 2013

[10] P. Rodrguez-Ayerbe and S. Olaru, On the disturbance model in the robustification of explicit predictive control, International Journal of Systems Science, vol 44(5), 2013.

[11] N. A. Nguyen, S. Olaru, G. Bitsoris and P. Rodriguez-Ayerbe, Explicit fragility margins for PWA control laws of discrete-time linear systems, Control Conference (ECC), European, Strasbourg, 2014, pp. 1450-1455, 2014.

[12] P. N. Efstratios, N. P. Faísca, K. I. Kouramas, P. Christos, Explicit robust model predictive control, In Proceedings of the international symposium on advanced control of chemical processes, ADCHEM 09, 2009.

[13] Sheng Yunlong and Liu Bin and Su Hongye and Chu Jian, Robust model predictive control for constrained linear systems based on contractive set and multi-parameter linear programming, Systems, Man and Cybernetics, 2003. IEEE International Conference on, vol. 4, pp. 3073-3078, 2003. 